\title{
ESTUDIO EMPÍRICO DE ESTRATEGIAS DE ALIANZAS APLICADAS EN LA INDUSTRIA ASEGURADORA MEXICANA
}

\section{EMPIRICAL STUDY OF ALLIANCE STRATEGIES IMPLEMENTED IN THE MEXICAN INSURANCE INDUSTRY}

José Bernardo Medina- Castillo; Deyanira Bernal -Domínguez y Rubén Miranda- López Doctorando del programa del PNPC "Doctorado en Ciencias Administrativas" de la UNAM Profesor de asignatura "B" de la FCA UAS1. Doctora en Ciencias Sociales por la UAS Profesora e investigadora de Tiempo Completo de la Facultad de Contaduría y Administración de la UAS2. Doctorante en Estudios Fiscales del PNPC FCA-UAS CONACYT Profesor e Investigador de Tiempo Completo de la Facultad de Contaduría y Administración de la UAS3.

\section{RESUMEN}

El objeto de estudio de esta investigación en las empresas aseguradoras mexicanas, es la búsqueda de evidencia empíricas sobre la relación de las estrategias de alianzas y sus efectos sinérgicos en las empresas del sector asegurador mexicano mediante el uso de Tecnologías de la Información y Comunicaciones (TIC'S). Para ello se realiza un estudio empírico descriptivo de tipo transversal, que proporciona una instantánea de las variables de interés y sus relaciones en un momento. Para la medición se utiliza un instrumento estructurado y se mide la percepción de los directivos sobre las variables estudiadas. Los resultados arrojan relaciones de asociación significativas de las estrategias sobre alianzas comerciales y los canales de distribución por medio de la banca y el uso de internet.

Palabras claves: Sector asegurador, alianzas estratégicas, Tecnologías de Información y Comunicaciones (TIC'S).

\section{SUMMARY}

The study object of this research in insurance companies in Mexico, is the search for evidence empirical relationship strategies Information Technology and

Recibido: 04 de diciembre de 2011. Aceptado: 02 de marzo de 2012. Publicado como ARTÍCULO CIENTÍFICO en Ra Ximhai 8(3): $331-341$.
Communications (ICTS), strategies for partnerships and synergies in the companies in the insurance sector Mexico. For this, an empirical study of cross-sectional descriptive, providing a snapshot of the key variables and relationships in a moment. For the measurement using a structured instrument and measure the perception of managers on the variables studied. The results show significant partnership strategies on business alliances and channels of distribution through the banking and internet use.

Keywords: Insurance industry, strategic alliances, Information and Communication Technologies (ICTS).

\section{INTRODUCCIÓN}

No cabe duda que la globalización ha provocado desde finales de la década de 1980 importantes cambios estructurales en los mercados financieros. México ha dirigido sus políticas hacia modelos orientados al mercado, lo que ha implicado un amplio proceso de liberalización y desregulación en esta área.

En estas tres últimas décadas, el mercado asegurador mexicano ha experimentado cambios significativos, adaptándose a las 
circunstancias de un entorno generado por la liberalización y la desregulación. En este sentido, se observa un marco de mayor apertura, dinamismo y competencia que se ha reflejado en el número de competidores que integran el mercado asegurador. Se han implementando estrategias que implican la asociación entre empresas competidoras, cooperación entre empresas dentro y fuera del sector asegurador, fusiones, adquisiciones, afiliaciones de algunas empresas a grupos financieros y la salida de otras del mercado.

Actualmente las alianzas estratégicas son un factor en el ambiente de los negocios encontrándose en los escenarios corporativos cada vez con mayor frecuencia, motivadas por: disgregación vertical en la reducción de los ciclos de vida de los artículos, el crecimiento de las necesidades de inversión de capital, el deseo de aumentar la competitividad a través del aprendizaje organizacional y la reducción de costos de investigación y desarrollo, entre otras razones. (Spekman, Lynn y Thomas, 1998). Existiendo a la vez acuerdos de colaboración en una relación vertical como por ejemplo un centro de un canal de distribución, y por otra parte las alianzas estratégicas horizontales que comprenden asociaciones de investigación y desarrollo entre empresas que son casi iguales.

Los objetivos de una alianza deben ser claros y conocidos a cada nivel administrativo, para que se identifique su relación con los objetivos particulares de la empresa. Las alianzas requieren concertaciones, tolerancias, respeto y sobre todo equidad; habrá que saber distinguir entre un aliado y una subsidiaria y no esperar resultados equivalentes, ya que sobre los aliados no se ejerce control, se comparte al igual que las responsabilidades. Siendo necesario tomar en cuenta los problemas y dificultades que se ahorran los integrantes de una alianza al no tener que efectuar grandes inversiones en otra plaza o país para expandir sus operaciones al lograr nuevos mercados a merced de sus aliados.

La globalización hace esenciales a las alianzas, ya que, al unir esfuerzos representan un vehículo de valor en servicios orientados al cliente, puesto que al cliente le interesa el producto o servicio por sus características y calidad sin importarle quien lo diseñe, produzca, distribuya y venda, mucho menos le dará cuidado el país de su procedencia; al cliente le interesa la marca y los detalles sin importar quien los proporcione, así pues las alianzas vienen a proporcionar una forma de hacer negocios con mayores rendimientos al compartir costos que van desde la investigación y desarrollo hasta la entrega del producto o prestación del servicio. Por otro lado (Stuart, 2000) señala como razón de incorporarse a alianzas, el potencial para aprender de sus socios los puntos importantes de lo que son las alianzas, en primer instancia, la relación que se tiene con el socio. Los motivos por los que se forman alianzas, pueden ser resumidos por Kale, Singh y Perlmutter, (2000): ganar ventaja competitiva en los mercados, acceso o análisis de nuevas tecnologías y el know-how, mas allá de la frontera de las empresas, llegar a economías de escala y su alcance o compartir el riesgo o la incertidumbre con sus socios. La necesidad de hacer alianzas puede fundamentarse en lograr un crecimiento a nivel internacional, a través de cooperaciones con empresas de los países anfitriones, para el intercambio de recursos y facilitar su introducción a dicho mercado. Es indispensable, que queden acordados expresamente los beneficios tanto económicos como de estatus, que recibirán cada uno de los socios, lo cual da sentido al valor creado en la alianza para ambas 
empresas.

En caso de una estrategia de adquisición, es recomendable una alianza previa entre la empresa adquirente y la empresa objetivo que puede dar capital experimental y ayude a la adquirente a desarrollar estrategias y patrones de comportamiento que permitan un intercambio eficaz y eficiente de los recursos antes de la adquisición. Asimismo, ofrecen al comprador información sobre el valor actual de negocio de forma que puede negociar mejor su adquisición (Hoffman y Schaper-Rinkel, 2001). Cuando estas circunstancias se dan, podemos considerar que la alianza puede ser una buena alternativa estratégica a disposición de los directivos que quieran crecer.

La globalización y el desarrollo de las tecnologías de la información, así como la ampliación de los mercados han producido importantes cambios en la forma de competir en muchas industrias. Las fusiones/adquisiciones y las alianzas estratégicas son los principales instrumentos utilizados por las empresas para adaptarse a la nueva estructura de la competencia (Ortiz de Urbina, et al, 2008). Las barreras de crecimiento a las que se enfrentan las pequeñas y medianas empresas, como la falta de empleados cualificados, la falta de financiación, los costes y las dificultades para desarrollar mercado fuera, hacen más complicado que éstas opten con tanta frecuencia como las grandes empresas por la puesta en marcha de fusiones/adquisiciones (Zozaya González, 2007).

En este caso, las alianzas pueden ser utilizadas para adaptarse a los cambios del entorno en forma flexible y rápida, dado que las alianzas requieren de menor compromiso y menores necesidades de inversión comparada con otras estrategias de crecimiento como las fusiones y adquisiciones; las alianzas son como un paso intermedio antes de tomar una decisión definitiva sobre la conveniencia o no de poner en marcha una fusión o una adquisición. Cabe señalar que las fusiones/ adquisiciones y las alianzas estratégicas son los principales instrumentos utilizados por las empresas para adaptarse a la nueva estructura de la competencia (Ortiz de Urbina, et al, 2008).

El objeto de estudio de la presente investigación son las empresas aseguradoras mexicanas y el estudio sobre la relación de cómo las TICS inciden en el desempeño de estas empresas y en las formas de hacer negocios; así como hace posibles nuevas formas de cooperación y alianzas, configurando nuevas redes organizativas e interorganizativas.

Los objetivos específicos de la investigación son:

1. Analizar el tipo de alianzas estratégicas y el uso de TICS en las empresas aseguradoras en México.

2. Indagar los niveles de significancia entre las alianzas comerciales y el uso de TICS como canal de distribución.

3. Identificar la correlación lineal entre las alianzas estratégicas y el uso de TICS.

\section{MATERIALES Y MÉTODOS}

El método de investigación aplicado fue cuantitativo (ver cuadro 1). El estudio de campo se realizó utilizando un cuestionario como instrumento de medición estructurado (ver anexo 1), el cual se aplicó mediante correo electrónico a 27 directivos del sector asegurador (ver figura 1), como resultado del método de muestreo aleatorio simple, éste se calculó con base en el universo total de la población objeto de estudio, el cual estuvo compuesto por 100 directivos de la 
Asociación Mexicana de Instituciones de Seguros (AMIS).

Para el tratamiento de los datos se utilizó un programa estadístico denominado Dyane versión 4 (Diseño y Análisis de Encuestas para Investigación Social y de Mercados), que reúne los métodos y técnicas estadísticas más comunes en investigación social. El método estadístico aplicado en este trabajo de investigación fue el de análisis multivariante, la bondad de éste es que estudia el comportamiento de tres o más variables al mismo tiempo.

Para la descripción general del fenómeno se utilizaron las medidas de tendencia central (medias) y desviaciones (desviación estándar). Adicionalmente se utilizaron tabulaciones (tablas de contingencias) para describir las situación general del fenómeno, apoyado con gráficos (Histogramas).

Para la prueba de hipótesis se aplicaron tabulaciones cruzadas y el test de la Ji cuadrada de Pearson, para determinar el grado de relación o asociación existente entre las variables cruzadas (Santesmases, 2009).

Adicionalmente a la prueba anterior se realizaron análisis de correlación lineal que permiten profundizar en la explicación e indagar el sentido de asociación de las variables.

Hallazgos del estudio empírico sobre

\section{Cuadro 1. Ficha técnica}

\begin{tabular}{|c|c|}
\hline Tipo de investigación & Estudio descriptivo transversal \\
\hline Población & Directivos de compañías de seguros mexicanas \\
\hline Tamaño de la población & 100 compañías aseguradoras \\
\hline Tipo de muestreo & Aleatorio simple \\
\hline Intervalo de confianza & $95,5 \%$ \\
\hline Posesión de atributo p & 0,99 \\
\hline Tamaño de la muestra & 27 empresas \\
\hline Error de muestreo & $3,29 \%$ \\
\hline Software estadístico & $\begin{array}{l}\text { Diseño y análisis de encuestas para investigación social y de } \\
\text { mercados (Dyane versión 4) }\end{array}$ \\
\hline Instrumento de medición & $\begin{array}{l}\text { Encuesta Estructurada (80 variables), formulario electrónico } \\
\text { (Anexo 1) }\end{array}$ \\
\hline $\begin{array}{l}\text { Validación del instrumento de } \\
\text { medición }\end{array}$ & Coeficiente Alfa de Cronbach $=0.9309$ \\
\hline Recolección de datos & Vía correo electrónico \\
\hline Técnicas estadísticas & $\begin{array}{ll}\text { - } & \text { Medidas de tendencia central } \\
\text { - } & \text { Tabulación simple } \\
\text { - } & \text { Tabulación cruzada y test de Ji cuadrada de Pearson } \\
\text { - } & \text { Análisis de correlación lineal }\end{array}$ \\
\hline
\end{tabular}

La tabulación es una forma sencilla de describir los comportamientos o características de grupos sociales en función de los atributos u otras características de tales grupos.

Este Test permite averiguar si existe una diferencia significativa entre los valores esperados y los observados de un conjunto de datos, aplicado a una tabla de contingencia, permite determinar el grado de relación o asociación entre dos variables, pero no proporciona información sobre la dirección de la misma, la cual debe inferirse de los resultados contenidos en la tabla de contingencia.

El coeficiente de correlación lineal es una medida del grado y dirección de la asociación entre dos variables, puede tomar valores comprendidos entre -1 y 1 . Un valor 0 indica ausencia de correlación. Si el coeficiente es positivo expresa una covariación de las variables en el mismo sentido, y un coficiente negativo, lo contrario. 


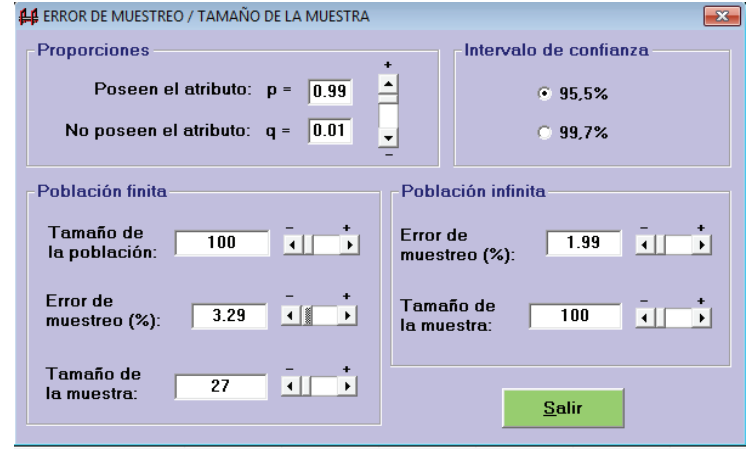

Figura 1. Error de muestreo y tamaño de muestra.

Fuente: Dyane, versión 4.

alianzas estratégicas del sector asegurador Las relaciones de asociación significativas de las alianzas estratégicas y el uso de las TICS, considerando que el objetivo de las alianzas son para obtener mayor cuota de mercado, diversificación, competitividad, motivaciones económicas, eficiencia en economías de escala o bien para crecimiento en las ventas; se observa la asociación más significativa con el uso de las TICS para mejorar la gestión y como elemento de fidelización de clientes (ver cuadro 2).

Las alianzas comerciales con instituciones bancarias para distribuir seguros (cuadro 3) se reflejan en el análisis de la relación con los canales bancarios para distribución de seguros, con el canal de distribución de seguros por internet y las alianzas con otros bancos. Las alianzas con distribuidores automotrices presentan una asociación con el uso de internet como estrategia de marketing y el uso de internet como canal de distribución. Las alianzas con el sector inmobiliario presentan una relación de asociación con el canal de distribución bancario y las alianzas con los mismos.

\section{Cuadro 2. Alianzas estratégicas y uso de TICS.}

\begin{tabular}{|c|c|c|c|c|}
\hline $\begin{array}{l}\text { No. } \\
\text { Var. }\end{array}$ & $\begin{array}{l}\text { Variable dependiente (A } \\
\text { explicar) o criterio }\end{array}$ & $\begin{array}{l}\text { No. } \\
\text { Var. }\end{array}$ & $\begin{array}{l}\text { Variable independiente (explicativa) } \\
\text { o predictora }\end{array}$ & $\begin{array}{l}\text { Grado de } \\
\text { significación }\end{array}$ \\
\hline 40 & $\begin{array}{l}\text { Alianzas estratégicas } \\
\text { para obtener una mayor } \\
\text { cuota de mercado }\end{array}$ & 13 & $\begin{array}{l}\text { Uso de Internet como medio para } \\
\text { mejorar la productividad }\end{array}$ & $\mathrm{p}=0,0200$ \\
\hline 41 & $\begin{array}{l}\text { Alianzas estratégicas } \\
\text { para diversificación }\end{array}$ & 11 & $\begin{array}{l}\text { Uso de Internet como estrategia de } \\
\text { marketing }\end{array}$ & $p=0,0348$ \\
\hline 42 & $\begin{array}{l}\text { Alianzas estratégicas } \\
\text { para competencia y } \\
\text { objetivos defensivos }\end{array}$ & 14 & $\begin{array}{l}\text { Uso de Internet como un medio para } \\
\text { mejorar la gestión }\end{array}$ & $\mathrm{p}=0,0151$ \\
\hline 43 & $\begin{array}{l}\text { Alianzas estratégicas por } \\
\text { motivaciones } \\
\text { económicas }\end{array}$ & 20 & Nivel de uso de TIC's & $\mathrm{p}=0,0305$ \\
\hline \multirow[t]{2}{*}{45} & \multirow{2}{*}{$\begin{array}{l}\text { Alianzas estratégicas } \\
\text { para mayor eficiencia } \\
\text { por economías de escala }\end{array}$} & 5 & $\begin{array}{l}\text { Uso de Internet como elemento de } \\
\text { fidelización de clientes }\end{array}$ & $p=0,0144$ \\
\hline & & 15 & Intercambio electrónico de datos & $\mathrm{p}=0,0229$ \\
\hline 46 & $\begin{array}{l}\text { Alianzas estratégicas } \\
\text { para crecimiento de } \\
\text { ventas }\end{array}$ & 15 & Intercambio electrónico de datos & $\mathrm{p}=0,0390$ \\
\hline
\end{tabular}

Fuente: investigación de campo. 
ESTUDIO EMPÍRICO DE ESTRATEGIAS DE ALIANZAS APLICADAS EN LA INDUS-

TRIA ASEGURADORA MEXICANA

\section{Cuadro 3. Alianzas comerciales y canales de distribución.}

\begin{tabular}{|c|c|c|c|c|}
\hline $\begin{array}{l}\text { No. } \\
\text { Var. }\end{array}$ & $\begin{array}{l}\text { Variable dependiente } \\
\text { (A explicar) o criterio }\end{array}$ & $\begin{array}{l}\text { No. } \\
\text { Var. }\end{array}$ & $\begin{array}{l}\text { Variable independiente (explicativa) } \\
\text { o predictora }\end{array}$ & Grado de significación \\
\hline \multirow[t]{2}{*}{28} & \multirow{2}{*}{$\begin{array}{l}\text { Alianzas comerciales } \\
\text { con instituciones } \\
\text { bancarias para distribuir } \\
\text { seguros }\end{array}$} & 72 & Canal de distribución (Bancos) & $\mathrm{p}=0.0002$ \\
\hline & & 74 & Canal de distribución (lnternet) & $\mathrm{p}=0.0069$ \\
\hline \multirow[t]{2}{*}{30} & \multirow{2}{*}{$\begin{array}{l}\text { Alianzas } \\
\text { distribuidores } \\
\text { automotrices }\end{array}$} & 11 & $\begin{array}{l}\text { Uso de internet como estrategia de } \\
\text { marketing }\end{array}$ & $\mathrm{p}=0.0078$ \\
\hline & & 74 & Canal de distribución (lnternet) & $\mathrm{p}=0.0133$ \\
\hline \multirow[t]{2}{*}{31} & \multirow{2}{*}{$\begin{array}{l}\text { Alianzas con el sector } \\
\text { inmobiliario }\end{array}$} & 72 & Canal de distribución (Bancos) & $\mathrm{p}=0.0207$ \\
\hline & & 75 & Alianzas con Bancos & $\mathrm{p}=0.0004$ \\
\hline \multirow[t]{3}{*}{32} & \multirow{3}{*}{$\begin{array}{l}\text { Alianzas con el sector } \\
\text { salud }\end{array}$} & 72 & Canal de distribución (Bancos) & $\mathrm{p}=0.0487$ \\
\hline & & 74 & Canal de distribución (lnternet) & $\mathrm{p}=0.0188$ \\
\hline & & 76 & Canal (Tiendas de autoservicios) & $\mathrm{p}=0.0056$ \\
\hline \multirow{3}{*}{34} & \multirow{3}{*}{$\begin{array}{l}\text { Alianzas con tiendas de } \\
\text { autoservicios }\end{array}$} & 71 & Oficinas propias & $\mathrm{p}=0.0204$ \\
\hline & & 74 & Canal de distribución (lnternet) & $\mathrm{p}=0.0374$ \\
\hline & & 75 & Alianzas con Bancos & $\mathrm{p}=0.0039$ \\
\hline \multirow{3}{*}{36} & \multirow{3}{*}{$\begin{array}{l}\text { Alianzas universidades } \\
\text { públicas y privadas }\end{array}$} & 72 & Canal de distribución (Bancos) & $\mathrm{p}=0.0024$ \\
\hline & & 73. & Canal de venta vía telefónica & $\mathrm{p}=0.0039$ \\
\hline & & 76 & Canal (Tiendas de autoservicios) & $\mathrm{p}=0.0066$ \\
\hline
\end{tabular}

Fuente: investigación de campo.

Las alianzas con el sector salud presentan una relación de asociación con el canal bancario, con el canal de distribución por internet y el canal de tiendas de autoservicio.

Respecto a las alianzas con tiendas de autoservicios, se tiene que presentan una relación significativa con algunas variables, como es el caso de la posesión de oficinas propias, el uso de internet como canal de distribución y las alianzas con bancos. Las alianzas con universidades públicas y privadas dependen en cierta manera de su relación con los canales bancarios de distribución, del canal de venta vía telefónica y del canal de tiendas de autoservicio.

A partir del análisis del cuadro 3, donde se concentran los resultados encontrados de la relación entre las estrategias de alianzas comerciales y canales de distribución bancarios o por internet; se analiza que existen relaciones de asociación significativas entre las alianzas comerciales tanto en canales de distribución bancarios como de internet.
Es preciso destacar que las de mayor significancia es el canal de distribución por bancos en las alianzas comerciales con instituciones bancarias para distribuir seguros con un grado de $p=0.0002$, alianzas con el sector inmobiliario y bancos con $\mathrm{p}=0.0004$ y alianzas universidades públicas y privadas con el canal de distribución bancario con $\mathrm{p}=0.0024$.

En cuanto a las alianzas que realizan las empresas aseguradoras para distribución de sus productos y servicios, aplicando un análisis de correlación lineal con las variables que inicialmente muestran una relación de asociación significativa mediante el test de Ji Cuadrada de Pearson, en el cuadro 4, se observa que las alianzas comerciales con instituciones bancarias para distribuir seguros posee un coeficiente de correlación importante con el canal de distribución de seguros por internet; las alianzas con empresas que administran tarjetas de crédito arrojan una correlación importante con las alianzas bancarias de igual manera las alianzas del sector inmobiliario. El sector 
Cuadro 4. Correlación lineal de alianzas y uso de TICS.

\begin{tabular}{|c|c|c|c|c|c|c|}
\hline \multicolumn{5}{|c|}{ Tabulación cruzada, Test de Ji Cuadrada de Pearson } & \multicolumn{2}{|c|}{$\begin{array}{l}\text { Correlación } \\
\text { Lineal Simple }\end{array}$} \\
\hline $\begin{array}{l}\text { No. } \\
\text { Var. }\end{array}$ & $\begin{array}{l}\text { Variable dependiente (A } \\
\text { explicar) o criterio }\end{array}$ & $\begin{array}{l}\text { No. } \\
\text { Var. }\end{array}$ & $\begin{array}{l}\text { Variable } \\
\text { (explicativa) } \\
\text { o predictora }\end{array}$ & $\begin{array}{l}\text { Grado de } \\
\text { significación } \\
\text { p }\end{array}$ & $\begin{array}{l}\text { Coeficient } \\
\text { e }\end{array}$ & $\begin{array}{l}\text { Grado de } \\
\text { significaci } \\
\text { ón } \\
\text { p }\end{array}$ \\
\hline 28 & $\begin{array}{l}\text { Alianzas comerciales } \\
\text { con instituciones bancarias } \\
\text { para distribuir seguros }\end{array}$ & 74 & Canal de distribución (lnternet) & $\mathrm{p}=0.0069$ & 0.5977 & 0.0009 \\
\hline 29 & $\begin{array}{llr}\text { Alianzas con empresas que } & \text { em } \\
\text { administran } & \text { tarjetas } \\
\text { crédito } & & \end{array}$ & 75 & Alianzas con Bancos & $\mathrm{p}=0.0030$ & 0.5956 & 0.0010 \\
\hline 31 & $\begin{array}{l}\text { Alianzas con el sector } \\
\text { inmobiliario }\end{array}$ & 75 & Alianzas con Bancos & $\mathrm{p}=0.0004$ & 0.4145 & 0.0312 \\
\hline & \multirow[b]{2}{*}{ Alianzas con el sector salud } & 72 & Canal de distribución (Bancos) & $\mathrm{p}=0.0487$ & 0.3987 & 0.0390 \\
\hline & & 74 & Canal de distribución (lnternet) & $\mathrm{p}=0.0188$ & 0.4015 & 0.0375 \\
\hline & \multirow{3}{*}{$\begin{array}{l}\text { Alianzas con tiendas de } \\
\text { autoservicios }\end{array}$} & 71 & Oficinas propias & $\mathrm{p}=0.0204$ & 0.3915 & 0.0430 \\
\hline & & 74 & Canal de distribución (lnternet) & $\mathrm{p}=0.0374$ & 0.5593 & 0.0023 \\
\hline & & 75 & Alianzas con Bancos & $\mathrm{p}=0.0039$ & 0.6310 & 0.0004 \\
\hline & $\begin{array}{l}\text { Alianzas con agencias de } \\
\text { viajes }\end{array}$ & 75 & Alianzas con Bancos & $\mathrm{p}=0.0054$ & 0.3806 & 0.0498 \\
\hline & \multirow{3}{*}{$\begin{array}{l}\text { Alianzas universidades } \\
\text { públicas y privadas }\end{array}$} & 72 & Canal de distribución (Bancos) & $\mathrm{p}=0.0024$ & 0.5626 & 0.0022 \\
\hline & & 73. & Canal de venta vía telefónica & $\mathrm{p}=0.0039$ & 0.4951 & 0.0085 \\
\hline & & 76 & Canal (Tiendas de autoservicios) & $\mathrm{p}=0.0066$ & 0.6043 & 0.0008 \\
\hline & $\begin{array}{llll}\begin{array}{l}\text { Alianzas con } \\
\text { funerario }\end{array} & & & \\
\end{array}$ & 76 & Canal (Tiendas de autoservicios) & $\mathrm{p}=0.0051$ & 0.3561 & 0.0678 \\
\hline & $\begin{array}{l}\text { Alianzas para mayor } \\
\text { eficiencia por economías de } \\
\text { escala }\end{array}$ & 15 & Intercambio electrónico de datos & $\mathrm{p}=0,0229$ & 0.5453 & 0.0032 \\
\hline
\end{tabular}

Fuente: investigación de campo.

salud para distribución de seguros, arroja un coeficiente de correlación significativo con el canal de distribución bancario y el canal de distribución por internet.

Las alianzas con tiendas de autoservicios para distribución de seguros poseen un coeficiente de correlación positivo con el canal de oficinas propias, el canal de distribución de internet y las alianzas con bancos. Las alianzas con agencias de viaje para distribución de seguros, se encuentra correlacionado de manera importante con las alianzas bancarias, de igual forma, las alianzas con universidades públicas y privadas para distribución de seguros, muestra una coeficiente de correlación importante con el canal de distribución bancario, el canal de distribución vía telefónica y canal de tiendas de autoservicios. Las alianzas con el sector funerario, presenta un nivel de correlación positivo con el canal de tiendas de autoservicio. Las alianzas para mayor eficiencia por economías de escala, muestran una correlación importante con el intercambio electrónico de datos.

\section{CONCLUSIONES}

Las alianzas comerciales con instituciones bancarias para distribución de seguros, se ven influenciadas positivamente por el uso 
del canal de distribución de seguros por internet, de igual manera, las alianzas de las aseguradoras con el sector inmobiliario para distribución de seguros, se benefician de manera importante con las alianzas bancarias. Por otro lado, las alianzas con el sector salud para distribución de seguros, se favorecen con el uso del canal de distribución bancario y el canal de distribución por internet.

Las tiendas de autoservicios para distribución de seguros se favorecen con el canal de distribución de seguros mediante oficinas propias, el canal de distribución de seguros por internet y las alianzas con bancos. Las agencias de viajes para distribución de seguros, dependen de manera importante de las alianzas bancarias, de igual forma, las alianzas con universidades públicas $\mathrm{y}$ privadas para distribución de seguros, muestran una dependencia importante con los canales de distribución bancaria, vía telefónica y canal de tiendas de autoservicios.

El sector funerario para distribución de seguros, presenta una correlación con el canal de tiendas de autoservicio. Las alianzas para mayor eficiencia por economías de escala, muestran una correlación importante con el intercambio electrónico de datos.

El uso de TICS mediante el canal de distribución de internet es importante en la mayoría de las alianzas estratégicas en el sector asegurador en México. Este tipo de análisis permite concluir que la aplicación de medios electrónicos incide en el mejor desempeño empresarial y en las nuevas formas de hacer negocio que permiten una mayor competitividad.

P. 1. ¿Su empresa posee actualmente una página $\mathrm{Web}$ ?

1. Si posee una ágina Web (Pasar a la pregunta 2)

2. No posee, pero tiene previsto desarrollarla en un plazo de un año (Pasar a la pregunta 17)

3. No posee, pero tiene previsto desarrollarla en un plazo mayor de un año (Pasar a la pregunta 17)

4. No posee, ni se ha planteado desarrollarla (Pasar a la pregunta 17)

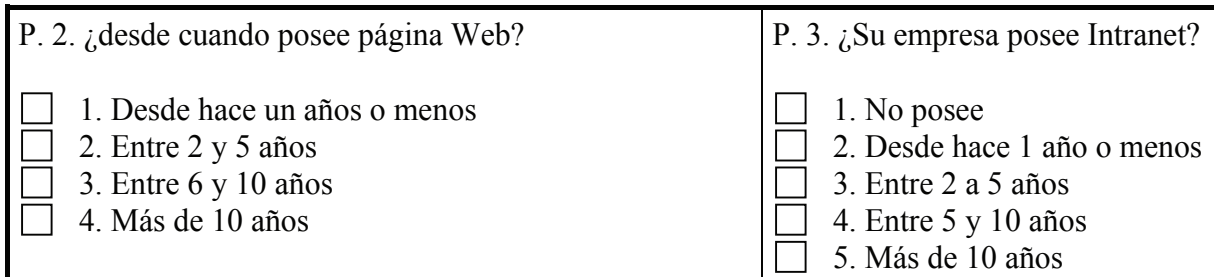

P. 4. La página Web cuenta con algunos de los siguientes servicios

1. Cotización en línea

2. Buzón de correo electrónico

3. Cotizació en línea

4. Solicitud de información personalizada

5. Presupuestos en línea

6. Venta en línea

7. Cobro en línea

8. Encriptación de datos para seguridad de las operaciones electrónicas 
¿En que medida utiliza su empresa la red de Internet?

\begin{tabular}{|l|l|l|l|l|l|}
\cline { 2 - 6 } \multicolumn{1}{c|}{} & Bajo & Medio bajo & Medio & Medio alto & Alto \\
\hline 5. Elemento de fidelización de clientes & $\square 1$ & $\square 2$ & $\square 3$ & $\square 4$ & $\square 5$ \\
\hline 6. Conocer mejor las necesidades de los clientes & $\square 1$ & $\square 2$ & $\square 3$ & $\square 4$ & $\square 5$ \\
\hline 7. Canal de venta o distribución & $\square 1$ & $\square 2$ & $\square 3$ & $\square 4$ & $\square 5$ \\
\hline 8. Para publicidad de sus productos & $\square 1$ & $\square 2$ & $\square 3$ & $\square 4$ & $\square 5$ \\
\hline 9. Como herramienta de comunicación & $\square 1$ & $\square 2$ & $\square 3$ & $\square 4$ & $\square 5$ \\
\hline 10. Como mejora de imagen & $\square 1$ & $\square 2$ & $\square 3$ & $\square 4$ & $\square 5$ \\
\hline 11. Como estrategia de marketing & $\square 1$ & $\square 2$ & $\square 3$ & $\square 4$ & $\square 5$ \\
\hline 12. Como oportunidad de mercado & $\square 1$ & $\square 2$ & $\square 3$ & $\square 4$ & $\square 5$ \\
\hline 13. Como medio para mejorar la productividad & $\square 1$ & $\square 2$ & $\square 3$ & $\square 4$ & $\square 5$ \\
\hline 14. Como un medio para mejorar la gestión & $\square 1$ & $\square 2$ & $\square 3$ & $\square 4$ & $\square 5$ \\
\hline 15. Intercambio electrónico de datos & $\square 1$ & $\square 2$ & $\square 3$ & $\square 4$ & $\square 5$ \\
\hline 16. Videoconferencias & $\square 1$ & $\square 2$ & $\square 3$ & $\square 4$ & $\square 5$ \\
\hline
\end{tabular}

¿En que grado considera que el uso de las TIC's influye en:?

\begin{tabular}{|l|l|l|l|l|l|}
\hline & Bajo & Medio bajo & Medio & Medio alto & Alto \\
\hline 17. Rendimiento en su empresa & $\square 1$ & $\square 2$ & $\square 3$ & $\square 4$ & $\square 5$ \\
\hline 18. Competitividad & $\square 1$ & $\square 2$ & $\square 3$ & $\square 4$ & $\square 5$ \\
\hline 19. Cambio en las estructuras organizativas & $\square 1$ & $\square 2$ & $\square 3$ & $\square 4$ & $\square 5$ \\
\hline
\end{tabular}

20. Nivel de uso de TIC's

\begin{tabular}{|l|l|l|l|l|}
\hline Bajo & Medio bajo & Medio & Medio alto & Alto \\
$\square 1$ & $\square 2$ & $\square 3$ & $\square 4$ & $\square 5$ \\
\hline
\end{tabular}

¿En que medida su empresa contrata servicios con empresas externas (Outsourcing)?

21. Outsourcing de servicios informáticos

22. Outsourcing de servicios financieros

23. Outsourcing de reparación de siniestros

24. Outsourcing de gestión de inversiones

25. Outsourcing de gestión de recursos humanos

26. Outsourcing de servicios generales

27. Outsourcing de otros servicios

\begin{tabular}{|l|l|l|l|l|}
\hline Bajo & Medio bajo & Medio & Medio alto & Alto \\
$\square 1$ & $\square 2$ & $\square 3$ & $\square 4$ & $\square 5$ \\
$\square 1$ & $\square 2$ & $\square 3$ & $\square 4$ & $\square 5$ \\
$\square 1$ & $\square 2$ & $\square 3$ & $\square 4$ & $\square 5$ \\
$\square 1$ & $\square 2$ & $\square 3$ & $\square 4$ & $\square 5$ \\
$\square 1$ & $\square 2$ & $\square 3$ & $\square 4$ & $\square 5$ \\
$\square 1$ & $\square 2$ & $\square 3$ & $\square 4$ & $\square 5$ \\
$\square 1$ & $\square 2$ & $\square 3$ & $\square 4$ & $\square 5$ \\
\hline
\end{tabular}

¿Su empresa tiene alianzas comerciales para venta y distribución de productos?

\begin{tabular}{|l|l|l|l|l|l|}
\hline $\begin{array}{l}\text { 28. Con instituciones bancarias para distribuir } \\
\text { seguros }\end{array}$ & $\square$ Bajo & Medio bajo & Medio & Medio alto & Alto \\
\hline $\begin{array}{l}\text { 29. Con empresas que administran tarjetas de } \\
\text { crédito }\end{array}$ & $\square 1$ & $\square 2$ & $\square 3$ & $\square 4$ & $\square 5$ \\
\hline 30. Con distribuidores automotrices & $\square 1$ & $\square 2$ & $\square 3$ & $\square 4$ & $\square 5$ \\
\hline 31. Sector inmobiliario & $\square 1$ & $\square 2$ & $\square 3$ & $\square 4$ & $\square 5$ \\
\hline 32. Sector salud & $\square 1$ & $\square 2$ & $\square 3$ & $\square 4$ & $\square 5$ \\
\hline 33. Call center & $\square 1$ & $\square 2$ & $\square 3$ & $\square 4$ & $\square 5$ \\
\hline 34. Tiendas de autoservicios & $\square 1$ & $\square 2$ & $\square 3$ & $\square 4$ & $\square 5$ \\
\hline
\end{tabular}




\begin{tabular}{|l|l|l|l|l|l|}
\hline 35. Agencias de viajes & $\square 1$ & $\square 2$ & $\square 3$ & $\square 4$ & $\square 5$ \\
\hline 36. Alianzas universidades públicas y privadas & $\square 1$ & $\square 2$ & $\square 3$ & $\square 4$ & $\square 5$ \\
\hline 37. Sector funerario & $\square 1$ & $\square 2$ & $\square 3$ & $\square 4$ & $\square 5$ \\
\hline 38. Otras entidades & $\square 1$ & $\square 2$ & $\square 3$ & $\square 4$ & $\square 5$ \\
\hline
\end{tabular}

\section{Razones para la realización de Alianzas} Estratégicas

\begin{tabular}{|l|l|l|l|l|l|}
\hline & Bajo & Medio bajo & Medio & Medio alto & Alto \\
\hline 39. Búsqueda de mayor ventaja competitiva & $\square 1$ & $\square 2$ & $\square 3$ & $\square 4$ & $\square 5$ \\
\hline 40. Obtener una mayor cuota de mercado & $\square 1$ & $\square 2$ & $\square 3$ & $\square 4$ & $\square 5$ \\
\hline 41. Diversificación & $\square 1$ & $\square 2$ & $\square 3$ & $\square 4$ & $\square 5$ \\
\hline 42. Competencia y objetivos defensivos & $\square 1$ & $\square 2$ & $\square 3$ & $\square 4$ & $\square 5$ \\
43. Motivaciones económicas & $\square 1$ & $\square 2$ & $\square 3$ & $\square 4$ & $\square 5$ \\
44. Maximización de utilidades & $\square 1$ & $\square 2$ & $\square 3$ & $\square 4$ & $\square 5$ \\
45. Mayor eficiencia por economías de escala & $\square 1$ & $\square 2$ & $\square 3$ & $\square 4$ & $\square 5$ \\
46. Crecimiento de ventas & $\square 1$ & $\square 2$ & $\square 3$ & $\square 4$ & $\square 5$ \\
\hline
\end{tabular}

P. 47. ¿Su empresa realiza alianzas estratégicas centradas en la participación institucional y que afecta al gobierno o propiedad de las empresas?

$\square$ 1. Participación con otras sociedades (dentro del negocio asegurador) en el mercado doméstico (Pasar a la pregunta 48)

2. Participación con otras sociedades (dentro del negocio asegurador) en el mercado exterior (Pasar a la pregunta 58)

$\square$ 3. Participación con otras sociedades fuera del negocio asegurador

4. Participación en consorcios

P. 48. ¿Su empresa cuenta con acuerdos de cooperación para la prestación de servicios de diferente naturaleza que afectan al negocio?

1. Con empresas prestadoras de servicios a clientes (servicios asistenciales, de prevención, etc.)

2. Con otras compañías de seguros para transferencia de riesgos

3. Con otras compañías de seguros para participar en redes de programas de coaseguro

4. Con otras compañías de seguros para la aceptación de reaseguro

5. Con otras compañías de seguros para la cesión de reaseguro

Nivel en el que considera se encuentra su empresa respecto a:

\begin{tabular}{|l|l|l|l|l|l|}
\hline & Bajo & Medio bajo & Medio & Medio alto & Alto \\
\hline 49. Atención al cliente & $\square 1$ & $\square 2$ & $\square 3$ & $\square 4$ & $\square 5$ \\
\hline 50. Atención al distribuidor & $\square 1$ & $\square 2$ & $\square 3$ & $\square 4$ & $\square 5$ \\
\hline 51. Orientación al trabajo en grupo & $\square 1$ & $\square 2$ & $\square 3$ & $\square 4$ & $\square 5$ \\
\hline 52. Aprendizaje contínuo & $\square 1$ & $\square 2$ & $\square 3$ & $\square 4$ & $\square 5$ \\
\hline 53. Satisfacción al cliente & $\square 1$ & $\square 2$ & $\square 3$ & $\square 4$ & $\square 5$ \\
\hline
\end{tabular}




\begin{tabular}{|c|c|c|c|c|c|}
\hline 54. Orientación a resultados & $\square 1$ & $\square 2$ & $\square 3$ & $\square 4$ & $\square 5$ \\
\hline 55. Capacidad de innovación & $\square 1$ & $\square 2$ & $\square 3$ & $\square 4$ & $\square 5$ \\
\hline $\begin{array}{l}\text { 56. Actualización de sus sistemas de } \\
\text { información }\end{array}$ & $\square 1$ & $\square 2$ & $\square 3$ & $\square 4$ & $\square 5$ \\
\hline 57. Imagen empresarial & $\square 1$ & $\square 2$ & $\square 3$ & $\square 4$ & $\square 5$ \\
\hline
\end{tabular}

P. 58. ¿Su empresa posee un sistema de Planificación de Recursos Empresariales (ERP)?

1. $\mathrm{Si}$

2. No

¿En que medida a contribuido el sistema ERP en los siguientes aspectos:?

\begin{tabular}{|c|c|c|c|c|c|}
\hline & Bajo & Medio bajo & Medio & Medio alto & Alto \\
\hline 59. Mejora de su imágen & $\square 1$ & $\square 2$ & $\square 3$ & $\square 4$ & $\square 5$ \\
\hline 60. Confiabilidad en la información del sistema & $\square 1$ & $\square 2$ & $\square 3$ & $\square 4$ & $\square 5$ \\
\hline 61. Tecnología de punta & $\square 1$ & $\square 2$ & $\square 3$ & $\square 4$ & $\square 5$ \\
\hline 62. Mejora del servicio al cliente & $\square 1$ & $\square 2$ & $\square 3$ & $\square 4$ & $\square 5$ \\
\hline 63. Mejora en los tiempos de respuesta & $\square 1$ & $\square 2$ & $\square 3$ & $\square 4$ & $\square 5$ \\
\hline 64. Reducción de costos operativos & $\square 1$ & $\square 2$ & $\square 3$ & $\square 4$ & $\square 5$ \\
\hline 65. Reducción del costo de calidad & $\square 1$ & $\square 2$ & $\square 3$ & $\square 4$ & $\square 5$ \\
\hline 66. Rápida adaptación a los cambios & $\square 1$ & $\square 2$ & $\square 3$ & $\square 4$ & $\square 5$ \\
\hline $\begin{array}{l}\text { 67. Integración de la información de las áreas } \\
\text { vitales }\end{array}$ & $\square 1$ & $\square 2$ & $\square 3$ & $\square 4$ & $\square 5$ \\
\hline 68. Competitividad de su empresa & $\square 1$ & $\square 2$ & $\square 3$ & $\square 4$ & $\square 5$ \\
\hline
\end{tabular}

\section{CANALES DE DISTRIBUCION}

\begin{tabular}{|l|l|l|l|l|l|}
\hline & Bajo & Medio bajo & Medio & Medio alto & Alto \\
\hline 69. Agencia & $\square$ 1 & $\square 2$ & $\square 3$ & $\square 4$ & $\square 5$ \\
\hline 70. Corredores & $\square 1$ & $\square 2$ & $\square 3$ & $\square 4$ & $\square 5$ \\
\hline 71. Oficinas propias & $\square 1$ & $\square 2$ & $\square 3$ & $\square 4$ & $\square 5$ \\
\hline 72. Bancos & $\square 1$ & $\square 2$ & $\square 3$ & $\square 4$ & $\square 5$ \\
\hline 73. Venta vía telefónica & $\square 1$ & $\square 2$ & $\square 3$ & $\square 4$ & $\square 5$ \\
\hline 74. Internet & $\square 1$ & $\square 2$ & $\square 3$ & $\square 4$ & $\square 5$ \\
\hline 75. Alianzas con Bancos & $\square 1$ & $\square 2$ & $\square 3$ & $\square 4$ & $\square 5$ \\
76. Tiendas de autoservicios & $\square 1$ & $\square 2$ & $\square 3$ & $\square 4$ & $\square 5$ \\
\hline 77. Otros canales & $\square 1$ & $\square 2$ & $\square 3$ & $\square 4$ & $\square 5$ \\
\hline
\end{tabular}

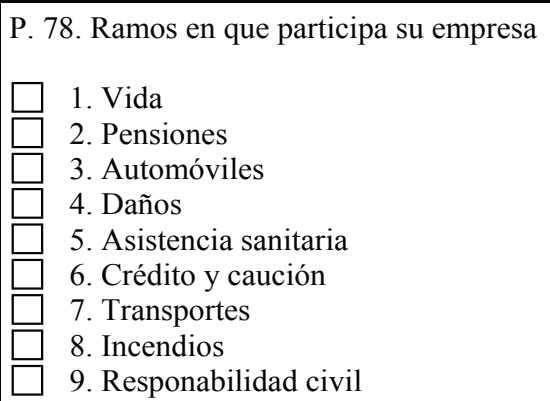

P. 79. Tiempo de antigüedad en el sector

$\square$ 1. 1-5 años

$\square$ 2. 6-10 años

3. 11-20 años

$\square$ 4. 21-40 años

5. Más de 40 años

P. 80. Nombre de la empresa 
Anexo 1. Cuestionario sobre el uso de Tecnologías de la Información y Comunicaciones (TICS)

La presente encuesta pretende investigar el uso de Tecnologías de la Información y Comunicaciones (TICS) del Sector Asegurador Mexicano. Sus respuestas son de gran interés para la investigación de Tesis Doctoral por lo que le agradezco tuviera la amabilidad de responderlas en cinco minutos de su valioso tiempo. Se garantiza el tratamiento anónimo de sus respuestas y así poder contribuir al conocimiento del Sector.

\section{LITERATURA CITADA}

Hoffmann, W.H.; Schaper-Rinkel, W. 2001. Acquire or Ally: A Strategy Framework for Deciding between Acquisition and Cooperation. Management International Review, Vol. 41, núm. 2, p. 131-159.

Kale, P., Singh, H. y Perlmutter, H. 2000. Learning and protection of proprietary assets in strategic alliances: building relational capital. Strategic Management Journal, 21/3, p. $217-237$.

Ortiz de Urbina Criado, M.; Montoro Sánchez, M.A.; Guerras Martín, L.A. 2008. Fusiones/ Adquisiciones y Acuerdos de Cooperación en la Unión Europea.

Santesmases, M. M. 2009. Dyane versión 4: Diseño y Análisis de Encuestas en Investigación Social y de mercado (incluye CD). Madrid: Editorial Pirámide.

Spekman, R., Forbes, T., Lynn, I. y MacAvoy, T. 1998. Alliance Management: a view from the past and a look to the future. Journal of Management studies, 32/6.

Stuart, T. 2000. Interorganizational alliances and the performance of firms: a study of growth and innovation rates in a high - technology industry. Strategic Management Journal, 21/8, p. 791 811.

Zozaya González, N. 2007. Las fusiones $\mathrm{y}$ adquisiciones como fórmula de crecimiento empresarial. Madrid: Documento de trabajo de la Dirección General de Política de la PYME.

José Bernardo Medina Castillo, Doctorando del programa del PNPC "Doctorado en Ciencias Administrativas" de la UNAM Profesor de asignatura "B" de la FCA UAS Profesorinvestigador "tiempo completo" del Instituto Tecnológico de Culiacán correo electrónico: jbernardo40@ gmail.com Cel: 6671544192

Deyanira Bernal Domínguez, Doctora en Ciencias Sociales por la UAS Profesora e investigadora de Tiempo Completo de la Facultad de Contaduría y Administración de la UAS. Correo electrónico: deyanirabernaldominguez@gmail. com, Celular: 6677757293

Rubén Miranda López, Doctorante en Estudios Fiscales del PNPC FCAUAS CONACYT Profesor e Investigador de Tiempo Completo de la Facultad de Contaduría y Administración de la UAS. Correo electrónico: fcarmirandagmail.com. Teléfono: 016672240599 